\title{
Helicopter Parenting Instrument: Estudos Psicométricos Iniciais com Adultos Emergentes
}

\author{
Helicopter Parenting Instrument: Initial Psychometric Studies with Emerging Adults
}

\author{
Daniela Borges ${ }^{1}$, Alda Portugal $^{2}$, Eunice Magalhães ${ }^{3}$, Luciana Sotero $^{4}$, Diogo Lamela $^{5}$ e Ana \\ Prioste $^{6}$
}

\section{Resumo}

O presente trabalho apresenta evidências de validade e fidelidade do Helicopter Parenting Instrument (HPI) no contexto português. Este instrumento avalia a perceção dos filhos sobre comportamentos parentais inapropriados face à sua etapa desenvolvimental (e.g., proteção excessiva, tomada de decisão pelos filhos). Participaram 187 adultos emergentes, com idades compreendidas entre os 18 e os 25 anos, respondendo a um questionário sociodemográfico, ao HPI, ao Inventário de Sintomas Psicopatológicos (BSI) para avaliar a sintomatologia ansiosa e depressiva; ao Inventário do Clima Familiar (ICF) para avaliar a coesão e o conflito familiares; e à Escala das Dimensões do Desenvolvimento Identitário (DIDS) para avaliar o desenvolvimento identitário. Evidências de validade de constructo foram obtidas através da análise fatorial confirmatória realizada, que revelou um modelo unidimensional ajustado aos dados, assim como de validade convergente a partir das correlações entre o HPI e BSI, ICF e DIDS. Foi também obtido um valor de consistência interna satisfatório.

Palavras-chave: parentalidade helicóptero, sintomatologia psicológica, clima familiar, desenvolvimento identitário, adultos emergentes

\begin{abstract}
The present study describes validity and reliability evidence of the Helicopter Parenting Instrument (HPI), in the Portuguese context. This instrument evaluates children's perception of inappropriate parental behaviors according to children's developmental stage (e.g., overprotection, decision-making by the children). A total of 187 emerging adults agreed to participate, aged between 18 and 25, answered a socio-demographic questionnaire, HPI, Brief Symptom Inventory (BSI) to assess anxiety and depression; the Family Climate Inventory (ICF) to assess cohesion and conflict, and the Identity Development Dimension Scale (DIDS) to assess identity development. Construct validity evidence was obtained through confirmatory factor analysis, showing an adequate fit to the one-dimensional model, as well as convergent validity evidence was found from the correlations between HPI, BSI, ICF and DIDS. A satisfactory internal consistency value was also found.
\end{abstract}

Keywords: helicopter parenting, psychological symptomatology, family climate, identity development, adults

\footnotetext{
${ }^{1}$ Mestrado em Psicologia Clínica. Escola de Psicologia e Ciências da Vida, Universidade Lusófona de Humanidades e Tecnologias, Lisboa, Portugal.

${ }^{2}$ Doutoramento em Psicologia Clínica. Professora Auxiliar. Universidade da Madeira, Portugal.

${ }^{3}$ Doutoramento em Psicologia. Investigadora Doutorada. Instituto Universitário de Lisboa (ISCTE-IUL), CIS-IUL, Lisboa, Portugal.

${ }^{4}$ Doutoramento em Psicologia Clínica. Professora Auxiliar. Faculdade de Psicologia e Ciências da Educação, Universidade de Coimbra, Portugal.

${ }^{5}$ Doutoramento em Psicologia Clínica, Professor Auxiliar. Universidade Lusófona do Porto, Porto, Portugal.

${ }^{6}$ Doutoramento em Psicologia Clínica. Professora Auxiliar. Escola de Psicologia e Ciências da Vida, Universidade Lusófona de Humanidades e Tecnologias, Lisboa. Faculdade de Psicologia, Universidade de Lisboa, Portugal. Correspondência: Escola de Psicologia e Ciências da Vida, Universidade Lusófona de Humanidades e Tecnologias, Campo Grande 376, 1749-024 Lisboa. Email: anaprioste@gmail.com

Revista Iberoamericana de Diagnóstico y Evaluación - e Avaliação Psicológica. RIDEP · N53 · Vol.4 · 33-48 · 2019

ISSN: 1135-3848 print /2183-6051online
} 


\section{Introdução}

A parentalidade engloba um conjunto de atividades desenvolvidas para assegurar a sobrevivência, a segurança e o desenvolvimento dos filhos (Hoghughi, 2004; Reader, Duncan, \& Lucey, 2005), no sentido de promover a autonomia e a socialização progressivas (Maccoby, 2000) e o domínio de ferramentas e estratégias para lidar com diversos desafios desenvolvimentais (e.g., físicos, económicos e psicossociais) (Bornstein, 2002). Os estudos na área da parentalidade têm sido desenvolvidos através de abordagens dimensionais, focando as práticas parentais (e.g., suporte emocional, rejeição e sobreproteção ou controlo físico e psicológico) (Darling \& Steinberg, 1993; Maccoby \& Martin, 1983; Steinberg, 2005), e tipológicas, enfatizando os estilos educativos parentais (autoritário, permissivo e autoritativo ${ }^{1}$ ) (e.g., Baumrind, 1996). A entrada no ensino superior da geração Millenium (i.e., a geração nascida após os anos 80) motivou o surgimento do conceito parentalidade helicóptero ${ }^{2}$ (Howe \& Strauss, 2003). A literatura tem descrito a parentalidade dos pais helicóptero através de níveis elevados de envolvimento, proteção, controlo e atenção dada aos filhos (Rainey, 2006). Por exemplo, os pais helicóptero tendem a comunicar frequentemente com e pelos filhos, intervir nos medos dos filhos, tomar decisões pelos filhos, investir o tempo pessoal nos objetivos dos filhos e remover os obstáculos e as barreiras que os filhos têm que enfrentar (LeMoyne \& Buchanan, 2011; Marano, 2004; Padilla-Walker \& Nelson, 2012; Segrin et al.,

\footnotetext{
${ }^{1}$ Os pais autoritários desenvolvem expetativas claras e firmes para os filhos, exigindo-lhes obediência, ordem e estrutura. Para além disso, este tipo de parentalidade caracteriza-se pelo uso da força, de ameaças, de restrições e de punições para moldar o comportamento dos filhos e de níveis baixos de suporte emocional (Baumrind, 1966). Os pais permissivos são apoiantes emocionalmente, não restringem nem modificam o comportamento dos filhos, sendo altamente recetivos (Baumrind, 1966). Os pais autoritativos são exigentes e recetivos, monitorizam o comportamento dos filhos de forma não intrusiva ou restritiva e estabelecem regras claras, sem serem punitivos (Baumrind, 1966).

${ }^{2}$ Este termo foi utilizado a primeira vez, em 1990, numa coleção de livros sobre parentalidade de Cline e Fay para designar pais hiperenvolvidos e protetores (Odenweller, Booth-Butterfield, \& Weber, 2014).
}

2012). Desta forma, estes comportamentos parentais têm sido perspetivados como uma forma de parentalidade excessiva, na qual os pais aplicam estratégias parentais inapropriadas à etapa desenvolvimental dos filhos, impedindo-os de assumir a responsabilidade das suas escolhas (Segrin et al., 2012). Contudo, estes comportamentos visam a garantia do sucesso dos filhos e a sua proteção de consequências potencialmente negativas (Segrin et al., 2012).

Tendo em conta a recência deste constructo, pela revisão de literatura efetuada, só se identificaram quatro instrumentos que avaliam a parentalidade helicóptero - Helicopter Parenting Scale (LeMoyne \& Buchanan, 2011), Helicopter Parenting Behaviors (Schiffrin et al. 2014), Helicopter Parenting Instrument (HPI; Odenweller et al., 2014) e Helicopter Parenting Scale (Montgomery's, 2010). Não estando nenhum destes instrumentos traduzidos e adaptados para o contexto português, o presente estudo centra-se no processo de adaptação do HPI à população portuguesa e no estudo inicial das propriedades psicométricas deste instrumento, com uma amostra de adultos emergentes portugueses com idades compreendidas entres os 18 e os 25 anos. Pretende-se, deste modo, contribuir para a expansão da literatura na área da parentalidade na adultez emergente em Portugal.

A pertinência deste estudo pode ser fundamentada através de vários argumentos. Entre eles, destacamos que, durante décadas, a investigação focada na parentalidade se centrou no impacto das práticas e dos estilos parentais nas trajetórias desenvolvimentais de crianças e adolescentes (Bradley-Geist \& Buchanan, 2014), negligenciando a sua relevância na etapa da adultez emergente. Por um lado, este facto poderá dever-se a recência do reconhecimento do período desenvolvimental que decorre entre os 18 e os 25 anos como uma etapa distinta da adolescência e da adultez (Arnett, 2004). Por outro lado, temos de considerar a tendência atual do prolongamento da coabitação em casa dos pais e o seu contributo na manutenção ou intensificação de funções e tarefas parentais após o fim da adolescência (Petrogiannis, 2011; Scabini, Marta, \& Lanz, 2006). Neste sentido, a literatura tem enfatizando o aumento da prevalência de comportamentos parentais específicos inadequados à etapa 
desenvolvimento dos filhos, nomeadamente a procura de contacto com professores para discutir as notas dos filhos (Bradley-Geist \& Buchanan, 2014). Com efeito, torna-se necessário compreender as implicações desenvolvimentais da parentalidade helicóptero, bem como informar, adequada e rigorosamente, a prática de diagnóstico e intervenção em contexto familiar e/ou parental.

Têm sido apontadas algumas limitações aos instrumentos existentes para avaliar a parentalidade helicóptero. Por exemplo, o Helicopter Parenting Scale (LeMoyne \& Buchanan, 2011) apresenta limitações concetuais e estatísticas e o Helicopter Parenting Behaviors (Schiffrin et al., 2014) avalia apenas a parentalidade helicóptero da mãe, excluindo o pai. Neste sentido, pela literatura revista, o HPI poderá revelar-se uma medida adequada. Realça-se também que o HPI enquadra-se numa perspectiva tipológica, em consonância com a literatura que sugere que a combinação de práticas parentais presentes neste tipo de parentalidade (i.e., níveis elevados de sobreprotecção e de suporte emocional, mas níveis baixos de apoio à autonomia dos filhos) a tornam uma tipologia parental distinta das propostas anteriormente (LeMoyne and Buchanan 2011; Padilla-Walker \& Nelson, 2012).

\section{Parentalidade helicóptero: Uma perspetiva ecológica}

Tendo como referencial teórico o Modelo

Ecológico de Desenvolvimento Humano (Bronfenbrenner, 1986), consideramos a influência de diversos contextos sistémicos no desenvolvimento da parentalidade helicóptero: macrossistémico (e.g., sistema social), microssistémico (e.g., contexto familiar) e cronossistémico (e.g., etapa desenvolvimental).

Ao nível macrossistémico, salienta-se a influência dos processos sociais (e.g., fatores sociais, económicos, políticos e culturais) na parentalidade helicóptero. Em termos globais, a existência de comportamentos parentais de hiperenvolvimento, proteção e controlo tornou-se particularmente evidente no contexto universitário, desde o início dos anos 2000, com entrada da geração Millenium no ensino superior. De relevar que esta geração é descendente da dos
Baby Boomers que priorizou a educação dos filhos como objetivo de vida (Gallo \& Gallo, 2001). Importa também considerar as alterações nos padrões sociais e culturais dos países industrializados que levaram a que as tarefas que marcavam o início da idade adulta, como o casamento e a parentalidade, fossem adiadas (Mendoça, Andrade, \& Fontaine, 2009). Foi neste contexto que surgiu a proposta da definição de uma nova etapa desenvolvimental - a adultez emergente (Arnett, 2004, 2015). Esta foi descrita através da experienciacão de um conjunto de processos psicológicos específicos: exploração da identidade (exploração de diferentes áreas da vida à medida que os adultos emergentes estabelecem compromissos e que se autodefinem); experimentação de possibilidades (exploração de diversas oportunidades e possibilidades); sentimento de ambiguidade (perceção ambígua de si, ou seja, a tendência de que os adultos emergentes não se sintam nem adolescentes nem adultos); negatividade (instabilidade e sobrecarga associadas aos desafios experienciados); e autocentração (autonomia e a liberdade pessoal) (Arnett, 2015; Baggio, Iglesias, Studer, \& Gmel, 2015; Gonçalves \& Barros, 2008). Em Portugal, bem como noutros países mediterrânicos, a inexistência de medidas sociais de apoio à autonomia dos adultos emergentes tende a reforçar os laços familiares, a acentuar o papel de suporte da família e a atrasar o processo de autonomização (Brandão, Saraiva, \& Matos, 2012; Guerreiro \& Abrantes, 2004), podendo também contribuir para a manutenção de uma parentalidade inadequada nesta etapa desenvolvimental dos filhos.

Ao nível microssistémico, realça-se a influência da família enquanto sistema mais influente ao longo de todo o ciclo de vida (Nogueira, 1998). A socialização e a individuação, enquanto funções centrais da família, permitem que os filhos se preparem para assumir papéis adultos nos âmbitos social, relacional, afetivo e profissional (Alarcão, 2000). Contudo, o prolongamento da coabitação com os pais durante a adultez emergente poderá promover a dependência em relação à família, dificultando o compromisso social e eternizando a condição de "adolescentes" (Jablonski \& Martino, 2013). A literatura na área da psicologia da família tem 
mostrado que as relações familiares com níveis elevados de fusão e de emaranhamento dificultam a diferenciação do self e o processo de individuação e autonomização (Bowen, 1976; Prioste, Tavares, \& Magalhães, Prioste, Tavares, \& Magalhães, 2019). Do mesmo modo, os comportamentos de hiperenvolvimento característicos de pais helicóptero estão associados a níveis baixos de autocontrolo e bemestar psicológico (Darlow, Norvilitis, \& Schuetze, 2017; Spokas \& Heimberg, 2009) e dificultam a transição para a idade adulta (Padilla-Walker \& Nelson, 2012; Ungar, 2009).

O cronossistema, referente à dimensão temporal, é um contexto transversal aos outros e, neste sentido, remete-nos para a adequação da parentalidade ao longo das etapas do ciclo vital. Apesar de a literatura mostrar que o envolvimento dos pais está associado positivamente ao ajustamento dos filhos (Fingerman, Cheng, Wesselman, Zarit, Fursterberg, \& Birditt, 2012; Gentzler, Oberhauser, Westerman, \& Nadorff, 2011; Gronlick \& Ryan, 1989; Hofer \& Moore, 2010; Kenyon \& Koerner, 2009), alguns estudos têm sugerido que a etapa desenvolvimental dos filhos poderá ter um efeito moderador nesta relação. Assim, na infância, níveis mais elevados de envolvimento e controlo estão associados positivamente ao ajustamento e desenvolvimento infantil; porém, na adultez emergente, o sentido desta relação é inverso (Segrin et al., 2015). Por exemplo, o estudo de Padilla-Walker e Nelson (2012), com uma amostra de 438 adultos emergentes com idades compreendidas entre os 18 e os 29 anos $\left(M_{\text {idade }}=19.65, D P=2.00\right)$, mostrou que participantes que percecionam os pais com níveis elevados de parentalidade helicóptero, tendem a considerar que os pais não lhes concedem o apoio e autonomia suficiente.

Ressalva-se, contudo, que a desadequação entre o nível desenvolvimental dos filhos e o excesso de envolvimento e sobreprotecção parentais pode ter efeitos igualmente negativos em etapas mais precoces do desenvolvimento (Borelli, Margolin, \& Rasmussen, 2015; Schiffrin, Godfrey, Liss, \& Erchull, 2015). Como a evidência empírica sugere que os comportamentos parentais são relativamente consistentes ao longo do tempo (e.g., Dallaire \& Weinraub, 2005; Forehand \& Jones, 2002), a sobreproteção parental em etapas desenvolvimentais precoces pode, eventualmente, prolongar-se sob a forma de parentalidade helicóptero na adultez emergente.

\section{Paradoxos da parentalidade helicóptero e associação a variáveis individuais, relacionais e familiares}

A parentalidade helicóptero pode implicar conselhos excessivos, resolução de problemas, apoio e suporte desnecessário e frequente, bem como, aversão parental ao risco, ansiedade parental e envolvimento no bem-estar emocional dos filhos (Segrin et al., 2015). Um dos paradoxos da parentalidade helicóptero centra-se no facto de ser motivada para assegurar resultados desenvolvimentais positivos para os filhos, apesar de a literatura mostrar, consistentemente, uma associação negativa entre este tipo de parentalidade e o ajustamento psicológico dos filhos (Segrin et al., 2015). Diversos estudos com adultos emergentes (e.g., Givertz \& Segrin, 2012; LeMoyne \& Buchanan, 2011; Schiffrin et al., 2014; Segrin et al., 2013; van Ingen et al., 2015) têm sugerido que níveis elevados de parentalidade helicóptero estão associados a níveis elevados de sintomatologia depressiva e ansiosa e de stress, e a níveis baixos de satisfação com a vida, autoaceitação e autoeficácia. A parentalidade excessiva tem também sido associada positivamente a traços de personalidade inadaptativos, nomeadamente, ao narcisismo (Locke et al., 2012; Segrin et al., 2012).

Este paradoxo tem sido explicado com base na teoria da autodeterminação (Deci \& Ryan, 2008; Ryan \& Deci, 2000), partindo da ideia de que o controlo parental excessivo viola os princípios básicos da autodeterminação autonomia, competência e relação - e contribuindo para o desajustamento. Deste modo, o hipercontrolo e envolvimento dos pais reduzem o sentido de autonomia (i.e., o sentimento de liberdade na tomada de decisão) e de competência (e.g., a confiança nas competências e capacidades) dos filhos, prejudicando também a relação paifilho (Schiffrin et al., 2013). Neste sentido, a literatura aponta para que a resolução dos problemas dos filhos pelos pais pode contribuir para a diminuição do sentido de competência e de autoeficácia dos filhos (Segrin et al., 2013) que, por sua vez, estão associados a níveis mais baixos 
de satisfação de vida (Meyer et al., 2007) e a níveis mais elevados de sintomatologia depressiva (Wei et al., 2005). O trabalho de LeMoyne e Buchanan (2011), focado na relação entre a parentalidade helicóptero e o bem-estar psicológico, com uma amostra de 317 adultos emergentes com idades compreendidas entre 18 e 25 anos, mostrou uma associação entre níveis elevados de parentalidade helicóptero e níveis inferiores de bem-estar psicológico e níveis superiores de consumo de ansiolíticos e antidepressivos.

Existem também evidências empíricas que mostram a associação entre a satisfação familiar e a parentalidade helicóptero. Por exemplo, o trabalho de Segrin e colaboradores (2012), com uma amostra de 477 pais de adultos emergentes $\left(M_{\text {idade }}=51.89, D P=6.10\right)$, sugeriu que os pais que se percecionavam como estando sobreenvolvidos na vida dos filhos tinham níveis mais baixos de satisfação familiar. Há ainda evidências que os pais que frequentemente tomam decisões $\mathrm{e}$ resolvem problemas pelos seus filhos em idade adulta emergente, tendem a limitar as oportunidades de estes desenvolverem as competências adequadas para serem bem sucedidos e autoconfiantes na vida adulta (PadillaWalker \& Nelson, 2012). O estudo de Luyckx, Soenens, Vansteenkiste, Goossens e Berzonsky (2007), como uma amostra de 565 estudantes adultos emergentes $\left(M_{\text {idade }}=18.7\right)$, mostrou que o controlo comportamental e psicológico parental está associado positivamente à ansiedade e à indecisão dos filhos, dificultando o seu processo de desenvolvimento da identidade com o aumento dos níveis de exploração ruminativa.

O sexo tem sido apontado como uma variável influente. No estudo de Reed, Duncan, LucierGreer, Fixelle e Ferraro (2016) verificou-se que as mães tendem a ter níveis de parentalidade helicóptero mais elevados com filhos do sexo masculino.

\section{O presente estudo}

Tendo em conta a relevância que o constructo de parentalidade helicóptero tem assumido nas comunidades académica e clínica, nos media e nas instituições escolares (Odenweller et al., 2014) e a inexistência de um instrumento validado para a população portuguesa que avalie estes comportamentos parentais inadequados à etapa desenvolvimental dos filhos, torna-se relevante a adaptação e a validação de uma medida. Deste modo, o presente estudo, através de um desenho quantitativo transversal, pretende: (1) traduzir e adaptar o HPI para a língua portuguesa; (2) analisar as propriedades psicométricas do HIP através da análise da validade (construto e convergente), da fidelidade (alfa de Cronbach e correlações médias interitens) e sensibilidade (assimetria, curtose, efeito chão e efeito teto); (3) analisar as diferenças entre sexos.

$O$ estudo da validade de constructo foi realizado através da exploração e da confirmação da estrutura fatorial da versão portuguesa do HPI numa amostra de adultos emergentes portugueses. Tendo em conta a literatura que mostra uma associação positiva entre a parentalidade helicóptero e a ansiedade e depressão (e.g., LeMoyne \& Buchanan, 2011; Schiffrin et al., 2012; Segrin et al., 2013), a validade convergente da escala foi estudada através da correlação entre o HPI, as medidas de depressão e ansiedade. Para além disso, a validade convergente foi estudada através da correlação entre o HPI e os processos de desenvolvimento da identidade propostos por Luyckx e colaboradores (2008), tendo em conta a literatura que indica que o controlo psicológico parental está associado positivamente à exploração ruminação e à exploração em amplitude (Luyckx et al., 2007). Por último, a validade convergente do HPI foi analisada através da correlação entre o HPI e a coesão e o conflito familiares, uma vez que a literatura indica que superproteção parental está associada positivamente a contextos familiares negativos e com níveis elevados de crítica e hostilidade (Segrin et al., 2015).

\section{Método}

\section{Participantes}

A amostra é constituída por 187 participantes, com idades compreendidas entre os 15 e os 25 anos $(M=21.20 ; D P=2.44)$, dos quais 121 eram do género feminino $(n=121 ; 64.7 \%)$ e 66 do género masculino $(n=66 ; 35.3 \%)$. Ao nível da escolaridade, $4.8 \%$ tinha entre 7 e 9 anos de escolaridade ( $n=9), 29.4 \%$ tinha entre 10 e 12 anos de escolaridade $(n=55), 57.8 \%$ frequentava o ensino superior $(n=108)$ e $8 \%$ tinha concluído o 
ensino superior $(n=15)$. No que concerne à zona de residência, $2.2 \%$ residia na zona norte de Portugal $(n=4), 0.5 \%$ residia no Alentejo $(n=1)$, $16.1 \%$ residia nos Açores $(n=30), 17.2 \%$ residia na Madeira $(n=32), 9.7 \%$ residia na zona centro $(n=18), 52.7 \%$ residia na zona da grande Lisboa $(n=98)$ e $1.6 \%$ residia noutra zona $(n=3)$.

No que diz respeito à coabitação, $56.1 \%$ da amostra coabitava com a família nuclear intacta $(n=105), \quad 11.2 \%$ coabitava com família monoparental $(n=21), 2.1 \%$ coabitava com família reconstituída $(n=4), 10.2 \%$ coabitava com família alargada $(n=19), 3.7 \%$ coabitava com a fratria $(n=7), 5.3 \%$ coabitava com amigos $(n=10), 4.3 \%$ coabitava com o par amoroso $(n=8)$ e $5.3 \%$ vivia sozinho/a $(n=10)$.

\section{Instrumentos}

Questionário de dados sociodemográficos. Os participantes responderam a um questionário de dados pessoais e sociodemográficos que incluía questões individuais (e.g., género, nacionalidade, idade, profissão, situação relacional, nível de escolaridade, zona residencial habitual) e questões sobre a família e a fratria (e.g., com quem viveu durante a infância e com quem vive atualmente, situação afetivo-relacional da mãe e do pai, número de irmãos biológicos e/ou adotivos e posição na fratria).

Investigating Helicopter Parenting (HPI; versão original: Odenweller et al., 2014). O HPI é uma escala de auto-relato constituída por 15 itens respondidos através de uma escala de Likert de sete pontos, de 1=discordo totalmente a $7=$ concordo totalmente. Este instrumento permite avaliar a perceção dos filhos em relação à parentalidade helicóptero, i.e., aos comportamentos parentais inadequados à etapa desenvolvimental dos filhos, nomeadamente sobreenvolvimento e protecção excessiva (e.g., "Às vezes os meus pais investem mais tempo e energia nas minhas coisas que eu próprio/a”). $\mathrm{O}$ instrumento original apresenta uma estrutura unidimensional e as pontuações mais elevadas na escala significam um nível mais elevado de parentalidade helicóptero.

No estudo de validação de Odenweller e colaboradores (2014), com uma amostra de 268 participantes com idades compreendidas entre 18 e 25 anos, a escala revelou um nível adequado de consistência interna $(\alpha=.78)$.

Inventário de Sintomas Psicopatológicos (Brief Symptom Inventory, BSI; versão original: Derogatis, 1982; tradução e adaptação para a população portuguesa: M. C. Canavarro, 1995). O BSI é um instrumento de auto-relato que avalia a sintomatologia psicológica. A tarefa do participante consiste na identificação da intensidade com que foi afetado, durante a última semana, por um conjunto de sintomas, utilizando para este efeito, uma escala de Likert de cinco pontos, de $0=$ nunca a $4=$ muitíssimas vezes. O BSI é composto por 53 itens que avaliam nove dimensões - Somatização, Obsessão-compulsão, Sensibilidade interpessoal, Depressão, Ansiedade, Hostilidade, Ansiedade fóbica, Ideação paranoide e Psicoticismo - e três índices globais que constituem avaliações sumárias de perturbação emocional: Índice geral de sintomas, Índice de sintomas positivos e Total de sintomas positivos (Canavarro, 1999).

No presente estudo, para estudar a validade convergente, foram utilizadas as dimensões Depressão e Ansiedade. A dimensão Depressão integra seis itens e avalia sintomas de afeto e humor disfórico, perda de energia vital, falta de motivação e de interesse pela vida (e.g., "Não ter interesse por nada") (Canavarro, 2007). A dimensão Ansiedade inclui seis itens e avalia sintomas de ansiedade e de ataques de pânico, bem como nervosismo e tensão (e.g., "Ter ataques de terror ou pânico") (Canavarro, 2007). As pontuações mais elevadas nas subescalas significam um nível mais elevado de intensidade da sintomatologia.

No estudo de validação realizado por Canavarro (1999), com uma amostra de 551 indivíduos, o BSI revelou níveis de consistência interna adequados entre $\alpha=.62$ para a dimensão Psicoticismo e $\alpha=.79$ para a dimensão Somatização. No presente estudo, as dimensões Ansiedade e Depressão revelaram também níveis de consistência interna adequados $(\alpha=.83$ e $\alpha=.87$, respetivamente).

Inventário do Clima Familiar (ICF; versão original: Teodoro, Allgayer, \& Land, 2009; versão portuguesa para investigação: Francisco, 2015). O ICF é uma medida de autorrelato composta por 22 itens e avalia o clima familiar através de uma 
escala Likert de cinco pontos, de 1=discordo completamente a 5=concordo completamente. Esta escala é constituída por quatro dimensões: Conflito, que integra seis itens que avaliam a relação agressiva, crítica e conflituosa entre os membros do sistema (e.g., "As pessoas criticamse umas às outras frequentemente"); Hierarquia, que incluiu seis itens que avaliam o poder e o nível do controle dentro da família (e.g., "Uns mandam e outros obedecem"); Apoio, integra cinco itens que avaliam o suporte material e emocional da família (e.g., "Procuramos ajudar as pessoas da nossa família quando percebemos que estão com problemas"); e Coesão, que contém cinco itens que avaliam o vínculo emocional entre os membros do sistema (e.g., "As pessoas gostam de passear e de fazer coisas juntas"). No presente trabalho foram apenas utilizadas as dimensões Conflito e Coesão. Pontuações mais elevadas nas dimensões significam um nível mais elevado de conflituosidade e coesão familiares.

No estudo de validação (Teodoro et al., 2009), com uma amostra de 276 participantes, as dimensões da escala mostraram níveis adequados de consistência interna, variando entre $\alpha=.84$ para a dimensão Conflito e entre $\alpha=.72$ para $a$ dimensão Hierarquia. No presente estudo, as dimensões Coesão e Conflito também apresentaram valores de consistência interna adequados ( $\alpha=.86$ e $\alpha=.89$, respetivamente).

Escala das Dimensões do Desenvolvimento Identitário (Dimensões of Identity Development Scale, DIDS; versão original: K. Luyckx, 2008; tradução e adaptação para a população portuguesa: Prioste, Lugar, Paulino, Jongenlenen, \& Rosa, 2018). A DIDS é uma medida de autorrelato composta por 25 itens que avaliam o desenvolvimento identitário através de uma escala de Likert de cinco pontos, de 1=discordo fortemente a 5=concordo fortemente. A DIDS avalia cinco dimensões: Exploração em profundidade, composta por cinco itens (e.g., "Falo com outras pessoas sobre os meus planos para o futuro") que medem a exploração de alternativas após a adesão a compromissos; Exploração em amplitude que integra cinco itens (e.g., "Estou a pensar em diferentes estilos de vida que podem ser bons para mim") que avaliam a exploração de alternativas prévias à adesão a compromissos; Compromisso, que inclui cinco itens (e.g., "Tenho uma imagem sobre o que vou fazer no futuro") que medem a adesão a compromissos; Identificação com o compromisso constituída por cinco itens (e.g., "Os meus planos para o futuro dão me auto-confiança") que avaliam o grau de segurança e de identificação em relação aos compromissos; e Exploração ruminativa composta por cinco itens (e.g., "Tenho dúvidas sobre o que quero realmente alcançar na vida") que avaliam a exploração progressiva de diversas alternativas e a não adesão a compromissos. Pontuações mais elevadas nas dimensões significam níveis mais elevados dos processos de desenvolvimento da identidade. No estudo de validação da DIDS (Luyckx et al., 2008), com uma amostra de adultos emergentes, as dimensões da escala mostraram níveis adequados de consistência interna, variando entre $\alpha=.79$ para a dimensão Exploração em profundidade e entre a $\alpha=.86$ para as dimensões Compromisso, Identificação com o compromisso e Exploração ruminativa. No estudo da validação da DIDS para a população portuguesa, com uma amostra de 285 participantes com idades compreendidas entre os 15 aos 29 anos, as dimensões da escala mostraram também níveis adequados de consistência interna, variando entre $\alpha=.65$ para a dimensão Exploração em profundidade e a $\alpha=.88$ para as dimensões Compromisso e Identificação com o Compromisso (Prioste et al., 2018). No presente estudo, as cinco dimensões revelaram também níveis de consistência interna aceitáveis, variando entre $\alpha=.63$ para a dimensão Exploração em Profundidade e $\alpha=.91$ para a dimensão Compromisso.

\section{Procedimento de recolha de dados}

A tradução do HPI foi realizada, individualmente, por duas psicólogas que dominam a língua inglesa. Após a tradução, procurou encontrar-se um consenso de modo a que os itens da versão portuguesa abarcassem o sentido dos itens da escala original, tendo em conta a equivalência lexical, conceptual e gramatical. De seguida, uma especialista em língua inglesa realizou a retroversão dos itens. A escala foi aplicada a um grupo-piloto de 20 participantes composto por adultos emergentes, com o qual foi discutida a adequação vocabular e a compreensão dos itens. A escala foi também 
analisada por duas especialistas na área da Psicologia da Família.

A recolha de dados iniciou-se após a aprovação do projeto de investigação pela Comissão de Ética e Deontologia em Investigação Científica da Escola de Psicologia e Ciências da Vida da Universidade Lusófona de Humanidade e Tecnologias. Foi recolhida uma amostra de 357 participantes entre outubro de 2017 e janeiro de 2018. Para inclusão neste estudo foram estabelecidos os seguintes critérios: ter idade compreendida entre os 18 e os 25 anos e ter nacionalidade portuguesa. Foram excluídos 88 participantes por não terem nacionalidade portuguesa $(n=88)$ e 82 por não cumprirem o critério etário estabelecido $(n=82)$. Uma amostra de 187 participantes cumpriu os critérios de inclusão estabelecidos, tendo sido integrada neste estudo.

A amostra recolhida foi de conveniência, tendo sido recolhida através de um procedimento não probabilístico, através da técnica "bola-deneve" por duas estratégias presenciais diferentes: (a) recolha de dados em grupo, no contexto de sala de aula a alunos do Ensino Superior (e.g., Universidade Lusófona de Humanidades e Tecnologias, Universidade de Lisboa, Universidade da Madeira e Universidade Católica Portuguesa); e (b) recolha de dados individual, através de contatos pessoais e sociais das investigadoras. Com o recurso a estas estratégias pretendeu-se que uma diversificação da amostra, incluindo adultos emergentes que frequentam e que não frequentam o ensino superior.

Durante a recolha da amostra, as investigadoras estiveram disponíveis para esclarecer as dúvidas relacionadas com as questões e/ou vocabulário. Após a explicitação dos objetivos do estudo, da garantia da confidencialidade, da possibilidade de desistência a qualquer momento e da assinatura do consentimento informado, os/as participantes colaboraram voluntariamente e sem remuneração.

\section{Procedimento de análise de dados}

Inicialmente, realizou-se a estatística descritiva dos 15 itens da versão portuguesa do HPI através do cálculo da média, desvio-padrão, máximo, mínimo, curtose, erro padrão da curtose, assimetria e erro padrão da assimetria. Para estudar a validade de constructo foi realizada uma análise fatorial confirmatória, com recurso ao software AMOS 23.0, tendo em conta a matriz de covariância e o estimador Máxima Verossimilhança (ML). Para analisar o ajustamento do modelo proposto foram utilizados os seguintes indicadores: o comparative fit índex (CFI); a razão do qui-quadrado pelos graus de liberdade $\left(\chi^{2} / \mathrm{gl}\right)$, o root meam square error of approximation (RMSEA) e o expected cross-validation índex (ECVI). De acordo com Marôco (2007), valores de CFI iguais ou superiores a .95 , valores de $\chi^{2} / \mathrm{gl}$ menores que cinco e valores de RMSEA iguais ou inferiores a .05 revelam um bom ajustamento do modelo. $\mathrm{O}$ índice de modificação (MI) foi utilizado para inclusão de parâmetros adicionais no modelo.

A análise das propriedades psicométricas da estrutura fatorial obtida (e.g., fiabilidade e validade convergente) e a análise de diferenças entre géneros foram realizadas com recurso ao software Statistical Package for the Social Sciences, versão 22 (SPSS). Para analisar a fidelidade da escala foi calculado o alfa de Cronbach $(\alpha)$ e as correlações médias interitens, rm.i.

Tal como já foi referido e, tendo em conta a literatura (e.g., Givertz et al., 2012; LeMoyne et al., 2011; Schiffrin et al., 2014; Segrin et al., 2012, 2013), a validade convergente foi estudada através do cálculo dos coeficentes de correlação de Pearson entre a pontuação do HPI e as dimensões Depressão Ansiedade do BSI, as dimensões Conflito e Coesão do ICF, e as dimensões Compromisso, Exploração em amplitude, Exploração ruminativa, Identificação com o compromisso e Exploração em profundidade da DIDS.

$O$ estudo das diferenças entre género foi realizado através do teste $t$-Student para amostras independentes, sendo que a potência dos resultados foi avaliada pelo $d$ de Cohen. Em todos os procedimentos estatísticos, foi estabelecido um nível de significância de 5\% ( $p<.05)$.

\section{Resultados}

\section{Estatística descritiva}

O Quadro 1 apresenta a estatística descritiva (média, desvio-padrão, assimetria, erro padrão da assimetria, curtose, erro padrão da curtose, mínimo e 
Quadro 1. Estatística Descritiva da Versão Portuguesa do HPI

\begin{tabular}{lcccccc}
\hline Item & $M(D P)$ & Assimetria (Erro) & $\begin{array}{c}\text { Curtose } \\
\text { (Erro) }\end{array}$ & Min-Max & $\begin{array}{c}\text { Efeito } \\
\text { Chão }(\%)\end{array}$ & $\begin{array}{c}\text { Efeito } \\
\text { Teto }(\%)\end{array}$ \\
\hline 1 & $3.62(2.01)$ & $.28(.18)$ & $-1.24(.36)$ & $1-7$ & 16.0 & 11.2 \\
2 & $3.85(1.92)$ & $.05(.18)$ & $-1.22(.36)$ & $1-7$ & 13.4 & 9.6 \\
3 & $2.02(1.37)$ & $1.27(.18)$ & $-.74(.36)$ & $1-7$ & 52.4 & 0.5 \\
4 & $3.28(1.63)$ & $.34(.18)$ & $-.90(.36)$ & $1-7$ & 14.4 & 1.6 \\
5 & $4.35(1.84)$ & $-.16(.18)$ & $-1.11(.36)$ & $1-7$ & 13.9 & 6.4 \\
6 & $2.63(1.52)$ & $.84(.18)$ & $.00(.36)$ & $1-7$ & 26.7 & 1.6 \\
7 & $2.81(1.81)$ & $.78(.18)$ & $-.46(.36)$ & $1-7$ & 31.0 & 4.8 \\
8 & $2.83(1.63)$ & $.57(.18)$ & $-.750(.36)$ & $1-7$ & 26.2 & 1.1 \\
9 & $3.90(1.81)$ & $-.23(.18)$ & $-1.03(.36)$ & $1-7$ & 15.5 & 5.3 \\
10 & $3.59(1.64)$ & $.11(.18)$ & $-.67(.36)$ & $1-7$ & 12.3 & 4.8 \\
11 & $3.09(1.74)$ & $.45(.18)$ & $-.92(.36)$ & $1-7$ & 22.5 & 2.7 \\
12 & $2.48(1.64)$ & $.94(.18)$ & $-.29(.36)$ & $1-7$ & 38.0 & 0.5 \\
13 & $5.65(1.56)$ & $-1.39(.18)$ & $1.26(.36)$ & $1-7$ & 2.7 & 35.3 \\
14 & $3.57(1.72)$ & $.46(.18)$ & $-.72(.36)$ & $1-7$ & 7.5 & 8.6 \\
15 & $4.13(1.73)$ & $-.25(.18)$ & $-.79(.36)$ & $1-7$ & 10.2 & 8.0 \\
\hline
\end{tabular}

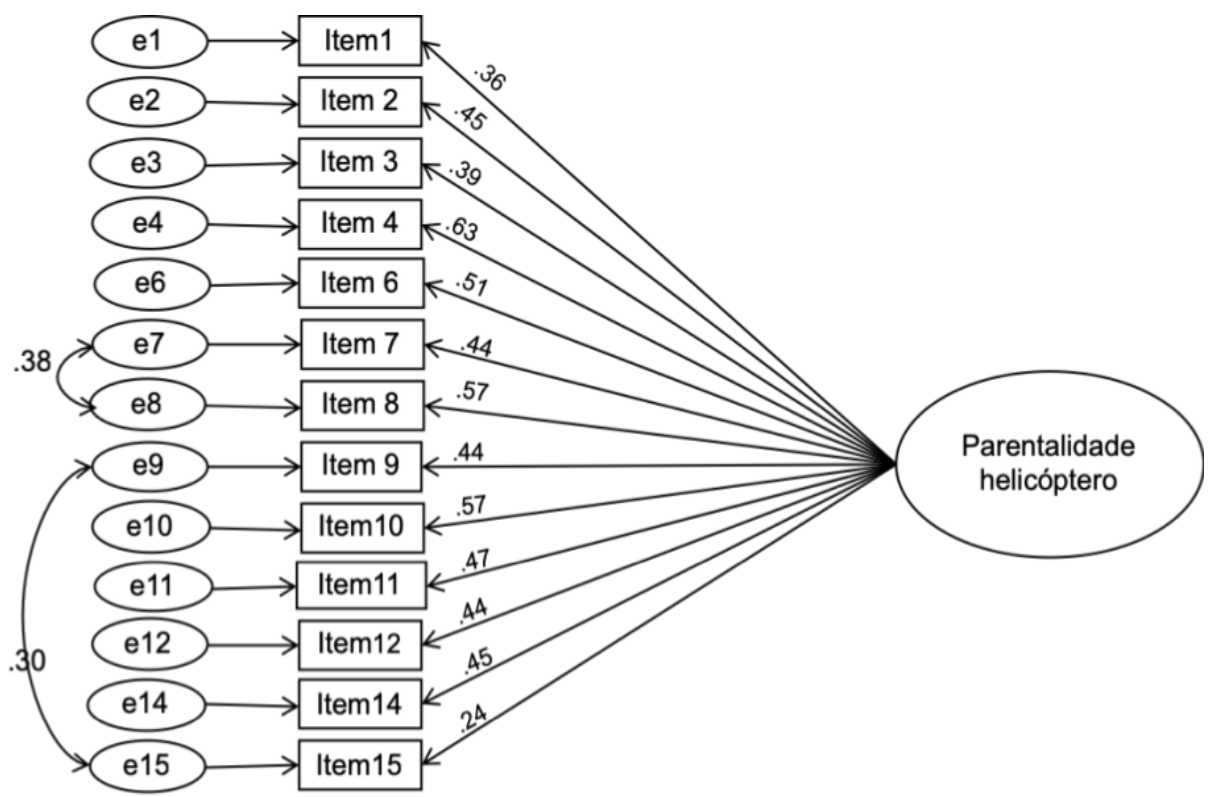

Figura 1. Estrutura fatorial do modelo unidimensional proposto e respetivos pesos fatoriais

máximo, efeito de teto e efeito de chão) dos itens da versão portuguesa do HPI.

No que concerne à assimetria, tal como se pode observar no Quadro 1, os itens 1, 3, 4, 6, 7, 8, 10, 11, 12, 14 apresentam uma distribuição assimétricos à direita, o item 2 apresenta uma distribuição simétrica e os itens 5, 9, 13 e 15 apresentam uma distribuição assimétrica à esquerda (Marôco, 2007). Relativamente ao achatamento, a maioria dos itens apresenta uma distribuição platicúrtica, com exeção do item 6 que apresenta uma distribuição mesocúrtica e o item 13 que apresenta uma distribuição leptocúrtica (Marôco, 2014).

\section{Validade de Constructo}

A validade de construto foi analisada através da confirmação da estrutura fatorial da versão portuguesa do HPI numa amostra de adultos portugueses. Para testar o modelo proposto foi conduzida uma análise fatorial confirmatória. $\mathrm{O}$ modelo proposto apresentou algumas fragilidades: $\chi^{2}(90)=234.87, p<.001, \chi^{2} / \mathrm{gl}=2.61, \mathrm{CFI}=.691 \mathrm{e}$ RMSEA=.093. Como o item 5 ("Os meus pais não interferem na minha vida a não ser que saibam que estou a passar por um problema físico ou emocional grave") e o item 13 ("Quando passo por uma situação difícil, os meus pais tentam sempre ajudar-me") apresentaram valores de saturação (Lambdas, $\lambda$ ) inaceitáveis $(b=.27 \mathrm{e}$ $b=.023$ ), procedeu-se a exclusão destes dois itens. Após a exclusão dos itens supracitados, o modelo apresentou índices de ajustamento mais adequados aos dados: $\chi^{2}(65)=125.80, \quad p<.001, \quad \chi^{2} / \mathrm{gl}=1.94$, $\mathrm{CFI}=.843, \quad \mathrm{RMSEA}=.071$ e ECVI $=1.096$. No entanto, a observação dos índices de modificação 
Quadro 2. Coeficientes de Correlação entre a Parentalidade Helicóptero e a Depressão, a Ansiedade, o Conflito, a Coesão, o Compromisso, a Exploração em Amplitude, a Exploração Ruminativa, a Identificação com o Compromisso e a Exploração em Profundidade $(\mathrm{N}=187)$

\begin{tabular}{lcccccccccc}
\hline Variáveis & 1 & 2 & 3 & 4 & 5 & 6 & 7 & 8 & 9 & 10 \\
\hline 1. Parentalidade Helicóptero & - & & & & & & & & & \\
2. Depressão & .06 & - & & & & & & & \\
3. Ansiedade & .05 & $.77^{* *}$ & - & & & & & & \\
4. Conflito & $.29^{* *}$ & $.29^{* *}$ & $.29^{* *}$ & - & & & & & \\
5. Coesão & $-.24^{* *}$ & $-.17^{*}$ & -.13 & $-.42^{* *}$ & - & & & & \\
6. Compromisso & -.13 & $-.28^{* *}$ & -.12 & .05 & .08 & - & & & \\
7. Exploração Amplitude & -.11 & $.16^{*}$ & $.22^{* *}$ & .06 & -.01 & .14 & - & & \\
8. Exploração Ruminativa & .11 & $.39^{* *}$ & $.31^{* *}$ & .03 & -.05 & $-.50^{* *}$ & $.29^{* *}$ & - & & \\
9. Identificação & -.10 & $-.24^{* *}$ & -.12 & -.00 & .08 & $.67^{* *}$ & .05 & $-.41^{* *}$ & - & \\
Compromisso & & & & & & & & & \\
10. Exploração Profundidade & -.08 & -.02 & .05 & -.12 & .06 & .14 & $.28^{* *}$ & $.25^{* *}$ & $.19^{*}$ & - \\
& & & & & & & & & &
\end{tabular}

Quadro 3. Médias e Desvios-padrão da Parentalidade Helicóptero para a Amostra Total e para o Sexo Feminino e Masculino

\begin{tabular}{lcccccccc}
\hline & \multicolumn{2}{c}{$\begin{array}{c}\text { Total } \\
(\mathrm{N}=187)\end{array}$} & \multicolumn{2}{c}{$\begin{array}{c}\text { Masculino } \\
(\mathrm{n}=66)\end{array}$} & \multicolumn{2}{c}{$\begin{array}{c}\text { Feminino } \\
(\mathrm{n}=121)\end{array}$} & $t$ & $p$ \\
\cline { 2 - 7 } & $M$ & $D P$ & $M$ & $D P$ & $M$ & $D P$ & & \\
\hline $\begin{array}{l}\text { Variável } \\
\text { Parentalidade helicóptero }\end{array}$ & 3.22 & .89 & 3.51 & 1.01 & 3.06 & .79 & 3.17 & .002 \\
\hline
\end{tabular}

(MIs) para as saturações (Lambdas, $\lambda$ ) e os erros de medida (Delta, $\delta$ ) indicou que seria aconselhável correlacionar os erros de medida dos seguintes itens: item 10 ("Os meus pais consideram-se bons pais quando resolvem um problema por mim.") e item 15 (“Os meus pais pensam que a sua tarefa é proteger-me de perigos e riscos."); e item 7 ("Os meus pais não se consideram bons pais quando não intervêm para me proteger de dificuldades") e item 8 ("Os meus pais sentem-se maus pais quando não tomo boas decisões"). De acordo com os MIs, adicionaramse dois parâmetros ao modelo, correlacionando os resíduos dos itens (item 10 -item 15 ; item 7 -item 8), atendendo à sua proximidade frásica (Marôco, 2010). Desta forma, a estrutura fatorial resultante permitiu obter melhores indicadores de ajustamento aos dados para o modelo proposto: $\chi^{2}(63)=88.14$, $p<.05, \quad \chi^{2} / \mathrm{gl}=1.40, \quad$ CFI $=.935, \quad \mathrm{RMSEA}=.046 \quad \mathrm{e}$ ECVI=.915. Na Figura 1 encontra-se apresentada a estrutura fatorial deste modelo.

\section{Validade Convergente}

De modo a avaliar a validade convergente da versão portuguesa do HPI, foram calculados os coeficientes de correlação de Pearson entre a parentalidade helicóptero, Ansiedade, Depressão,
Coesão, Conflito, Compromisso, Exploração em Amplitude, Exploração Ruminativa, Identificação com Compromisso e Exploração em Amplitude (vide Quadro 2).

Pela análise dos resultados, relativamente à validade convergente, observa-se que a EPH se encontra associado significativa e positivamente à dimensão Conflito e negativamente à dimensão Coesão familiar, sendo ambas as associações fracas.

\section{Fidelidade}

O alfa de Cronbach $(\alpha)$ e homogeneidade (correlação média interitens foram calculados e revelaram uma consistência interna adequada da escala: $\alpha=.78, \mathrm{rm} . \mathrm{i}=.22$.

\section{Análise das diferenças de sexo}

No Quadro 3 apresenta-se a média e o desviopadrão da parentalidade helicóptero para a amostra total, sexo feminino e masculino. A análise das diferenças de sexo mostrou que existe uma diferença estatisticamente significativa, indicando que os participantes do sexo masculino percebem os seus pais com níveis mais elevados de parentalidade helicóptero, em relação ao sexo feminino, $t(106.5)=3.17, p=.002, d=.50$. 


\section{Discussão}

O HPI é um instrumento de autorrelato que avalia a perceção dos filhos em relação à parentalidade helicóptero, isto é, aos comportamentos parentais inadequados à etapa desenvolvimental dos filhos, nomeadamente o sobreenvolvimento e a protecção excessiva. Este trabalho pretendeu traduzir e adaptar para a língua portuguesa o HPI e estudar as propriedades psicométricas deste instrumento. Especificamente, foi analisada a validade de constructo e a fidelidade da medida, numa amostra de adultos emergentes portugueses (18-25 anos). Foram também analisadas as diferenças da parentalidade helicóptero em função do sexo. Este estudo teve como finalidade contribuir para o enriquecimento da literatura na área da parentalidade na adultez emergente em Portugal, através da supressão de algumas das lacunas encontradas na literatura, nomeadamente, a inexistência de instrumentos adaptados para a população portuguesa que avaliem a parentalidade helicóptero.

No que diz respeito à validade de construto, como a AFC revelou, inicialmente, que os itens 5 e 13 apresentavam valores de saturação inaceitáveis, foram excluídos. A AFC final do HPI relevou um bom ajustamento à estrutura da variância-covariância observada entre os 13 itens, embora alguns itens (e.g., itens 1 e 3 ) tenham apresentado pesos fatoriais padronizados mais baixos $(\lambda<.4)$. As correlações observadas entre os erros dos itens podem estar relacionadas com a similitude na formulação ou no conteúdo dos itens (Marôco, 2010).

Relativamente à validade convergente, não se observaram associações significativas entre a parentalidade helicóptero, a ansiedade, a depressão e os cinco processos de desenvolvimento da identidade, o que não corrobora os resultados de estudos anteriores (Givertz \& Segrin, 2012; LeMoyne \& Buchanan, 2014; Luyckx et al., 2007; Schiffrin et al., 2014; Segrin et al., 2013). Estes resultados podem ser explicados atendendo ao facto do HPI avaliar a parentalidade helicóptero dos pais no global. Supomos que, se se tivesse analisado, individualmente, a parentalidade da mãe e do pai, em consonância com outros instrumentos que avaliam a parentalidade (e.g., EMBU-C
[Canavarro \& Pereira, 2007]), poder-se-iam encontrar associações significativas entre as variáveis de interesse. Estes resultados podem também ser explicados atendendo a características macrossistémicas, nomeadamente, à tendência atual do prolongamento da coabitação em casa dos pais, à intensificação de funções e tarefas parentais nesta etapa desenvolvimental (e.g., Brandão et al., 2012; Guerreiro \& Abrantes, 2004; Petrogiannis, 2011; Scabini, Marta, \& Lanz, 2006) e ao aumento da prevalência de comportamentos parentais específicos inadequados à etapa desenvolvimento dos filhos (Bradley-Geist \& Buchanan, 2014). Estes factores poderão contribuir para uma "normalização" de comportamentos parentais do tipo helicóptero. Não sendo estes comportamentos experienciados pelos pais nem pelos filhos adultos emergentes como inadequados, poderão não reflectir-se no ajustamento psicológico dos filhos. Por exemplo, num estudo realizado em Portugal, mostrou que as práticas parentais dos pais autoritários portugueses não são percebidas tão negativamente como nos Estados Unidos da América ou noutros países europeus (Fontaine, 1990). Em Portugal, as práticas parentais de sobreprotecção tendem a ser percebidas como uma dimensão normal da função educativa dos pais (Montandon, 2005).

É também plausível supor que o surgimento de sintomatologia ansiosa e depressiva poderá também estar associada a outras variáveis não incluídas neste estudo, nomeadamente, o tempo de exposição a comportamentos parentais inadequados à etapa desenvolvimento dos filhos, características das trajectórias desenvolvimentais (e.g., saída de casa dos pais para viver de forma independente). Para além disso, o surgimento de sintomatologia ansiosa e depressiva pode ser precipitado pela sua associação a outros eventos de vida relevantes (e.g., projetos de vida associados a uma relação afetiva) e/ou pode expressar-se, diferentemente, a longo prazo e, como tal, não ser observável num estudo de natureza transversal.

Ainda em relação à validade convergente, os resultados mostraram que a parentalidade helicóptero se associa significativamente ao Conflito e à Coesão familiar, o que corrobora os estudos de estudos anteriores (Koerner \& Fitzpatrick, 2002; Odenweller et al., 2014; Segrin 
et al., 2012, 2015). Para além disso, este resultado apoia os resultados do trabalho realizado por Segrin e colaboradores (2012) que mostraram uma associação negativa entre os níveis de parentalidade helicóptero e os níveis de satisfação familiar. Apesar de os dados obtidos não suportarem o paradoxo referido anteriormente [i.e., a motivação subjacente aos comportamentos parentais tipo helicóptero é assegurar resultados desenvolvimentais positivos para os filhos (Segrin et al., 2015), garantindo a sua protecção, mas a evidência empírica mostra uma relação negativa entre os níveis de parentalidade helicóptero e o ajustamento dos filhos (Givertz \& Segrin, 2012; LeMoyne \& Buchanan, 2011; Schiffrin et al., 2014; Segrin et al., 2013)], é plausível supor que o facto de a parentalidade helicóptero se associar positivamente à agressividade, crítica e conflito familiares e negativamente ao vínculo emocional existente entre elementos da família poderá ser lido como um paradoxo. Neste sentido, os comportamentos parentais de sobreenvolvimento, a sobreproteção e o hipercontrolo potenciam a desconexão e desligamento dos filhos à família, aumentando a hostilidade nas dinâmicas familiares.

No que diz respeito à fidelidade, a versão portuguesa do EPH mostrou possuir valores de consistência interna aceitáveis, à semelhança do estudo da versão original de Odenweller e colaboradores (2014). O facto de os adultos emergentes percepcionarem os pais com níveis mais elevados de parentalidade helicóptero vai ao encontro dos resultados do estudo de Reed e colaboradores (2016).

Sendo este estudo um contributo inicial para o estudo das propriedades psicométricas da EPH, apresenta limitações que restringem a sua validade: a amostra foi selecionada através de uma técnica de amostragem não probabilística, denominada de "bola de neve" e, pelo facto de não ser representativa da população portuguesa, os resultados têm um carácter exploratório. A amostra é maioritariamente constituída por estudantes universitários do género feminino, o que limita também a possibilidade de generalização dos resultados deste trabalho. Outras limitações relevantes prendem-se com o facto de não terem sido estudadas a estabilidade temporal do instrumento (teste-reteste) e de não ter sido estudada a validade discriminante. Em relação à validade convergente, pesa como limitação o facto de esta não ter sido avaliada através da correlação entre o HPI e outro instrumento que meça um construto semelhante.

Os estudos futuros com o HPI deverão ser desenvolvidos de forma a superar as limitações apontadas. Será necessário também replicar este estudo com amostras mais equitativas em termos da distribuição por sexo. Os trabalhos futuros também deverão contribuir para o processo de validação do HPI, analisando a validade convergente através da correlação com outro que avalie um construto similar, por exemplo, o Helicopter Parenting Behaviors (Schiffrin et al. 2014) ou o Helicopter Parenting Scale (LeMoyne \& Buchanan, 2011). Seria também importante realizar um estudo longitudinal para analisar, não só a estabilidade temporal do HPI, como também para perceber, de forma mais aprofundada, de que forma os níveis de parentalidade vão evoluindo ao longo do tempo na adultez emergente.

Considera-se também importante analisar, em estudos futuros, a parentalidade helicóptero, tendo por base a teoria da autodeterminação (Deci \& Ryan, 2008; Ryan \& Deci, 2000) e incluindo as variáveis autonomia, competência e relação paifilho (Schiffrin et al., 2013). Por último, seria importante explorar mais aprofundadamente a leitura paradoxal dos resultados proposta em relação aos comportamentos de parentalidade helicóptero potenciarem a desconexão e desligamento dos filhos à família. A parentalidade helicóptero será uma forma de parentalidade excessiva inadaptada face à etapa desenvolvimental dos filhos ou poderá também ser uma estratégia parental paradoxal para incentivarem os filhos a percorrerem um caminho centrífugo e de autonomização em relação à família?

Apesar das limitações apontadas, o HPI pode constituir-se um instrumento de avaliação da parentalidade helicóptero, produzindo dados fiáveis e válidos, podendo ser utilizados por clínicos e por investigadores, em amostras de adultos emergentes. 


\section{Referências}

Alarcão, M. (2000). (Des)Equílibrios Familiares $\left(1^{a}\right.$ ed.). Coimbra: Quarteto.

Alsaker, F. D., \& Flammer, A. (2006). Pubertal maturation. In S. Jackson \& L. Goossens (Eds.), Handbook of adolescent development (pp. 30-50). Hove and New York: Psychology Press.

Arnett, J. J. (2004). Emerging adulthood: The winding road from the late teens through the twenties. New York, NY: Oxford University Press.

Baggio, S., Iglesias, K., Studer, J., \& Gmel, G. (2015). An 8-item short form of the inventory of dimensions of emerging adulthood (IDEA) among young Swiss men. Evaluation \& The Health Professions, 38(2), 246-254.

Baumeister, R. F., Shapiro, J. P., \& Tice, D. M. (1985). Two kinds of identity crisis. Journal of Personality, 53, 407-424.

Baumrind, D. (1966). Effects of authoritative control on child behavior. Child Development, 37, 887-907.

Bornstein, M. (2002). Preface. In M. H. Bornstein (Ed.), Handbook of parenting (Vol. 3, pp. xvxvii). New Jersey: Lawrence Erlbaum Associates.

Bowen, M. (1976). Theory in the practice of psychotherapy. In P. J. Guerin (Ed.), Family therapy: Theory and practice (pp. 42-90). New York: Gardner.

Brandão, T., Saraiva, L., \& Matos, P. M. (2012). $\mathrm{O}$ prolongamento da transição para a idade adulta e o conceito de adultez emergente: Especificidades do contexto português e brasileiro. Análise Psicológica, 30(3), 301313.

Borelli, J. L., Margolin, G., \& Rasmussen, H. F. (2015). Parental overcontrol as a mechanism explaining the longitudinal association between parent and child anxiety. Journal of Child and Family Studies, 24, 1559-1574. doi:10.1007/s10826-014-9960-1

Bronfenbrenner, U. (1986). Ecology of the family as a context for human development: Research perspectives. Developmental Psychology, 22(6), 723-742. doi:10.1037/0012-1649.22.6.723.
Canavarro, M. C. (1999). Inventário de Sintomas Psicopatológicos-BSI. In Mário R. Simões, M. Gonçalves \& L. S. Almeida (Eds.), Testes e Provas Psicológicas em Portugal (Vol.2, pp. 1-27). Braga: APPORT/SHO.

Canavarro, M. C. (2007). Inventário de Sintomas Psicopatológicos: Uma revisão crítica dos estudos realizados em Portugal. In M. Simões, C. Machado, M. Gonçalves, \& L. Almeida (Eds.), Avaliação psicológica: Instrumentos validados para a população Portuguesa (vol. III, pp. 305-331). Coimbra: Quarteto Editora.

Canavarro, M. C., \& Pereira, A. I. (2007). A percepção dos filhos sobre os estilos educativos parentais: A versão portuguesa do EMBU-C. Revista Iberoamericana de Diagnóstico y Evaluación - e Avaliação Psicológica, 24(2), 193-210.

Barroso, R., \& Machado, C. (2011). Definições, dimensões e determinantes da parentalidade. Psychologica, 52, 211-230.

Dallaire, D. H., \& Weinraub, M. (2005). The stability of parenting behaviors over the first 6 years of life. Early Childhood Research Quarterly, 20(2), 201-219. doi:10.1016/j.ecresq. 2005.04.008.

Darling, N., \& Steinberg, L. (1993). Parenting Style as Context: An Integrative Model. Psychological Bulletin, 113(3), 487-496.

Darlow, V., Norvilitis, J. M., \& Schuetze, P. (2017). The relationship between helicopter parenting and adjustment to college. Journal of Child and Family Studies, 26, 2291-2298. doi:10.1007/s10826-017-0751-3

Deci, E. L., \& Ryan, R. M. (2008). Facilitating optimal motivation and psychological wellbeing across life's domains. Canadian Psychology, 49, 14-23. doi:10.1037/0708-5591.49.1.14

Bradley-Geist, J., \& Buchanan, J. (2014). Helicopter parents: An examination of the correlates of over-parenting of college students. Education, Training, 56(4), 314-328.

Fingerman, K. L., Cheng, Y., Wesselmann, E. D., Zarit, S., Furstenberg, F., \& Birditt, K. S. (2012). Helicopter parents and landing pad kids: Intense parental support of grown children. Journal of Marriage and Family, 74, 880-896. doi:10.1111/j.1741-3737.2010.00987.x. 
Fontaine, M. (1990). Pratiques éducatives familiales et motivation pour la réussite d'adolescents en function du context social. In M. Montandon (Ed.), As práticas educativas parentais e a experiências das crianças. Educação \& Sociedade, 26(91), 485-507.

Forehand, R., \& Jones, D. J. (2002). The stability of parenting: A longitudinal analysis of innercity African-American mothers. Journal of Child and Family Studies, 11(4), 455-467. doi:10.1023/ A:1020935525335

Gallo, E., \& Gallo, J. J. (2001). Silver spoon kids: How successful parents raise responsible children. Chicago, IL: McGraw-Hill.

Gentzler, A. L., Oberhauser, A. M., Westerman, D., \& Nadorff, D. K. (2011). College students' use of electronic communication with parents: Links to loneliness, attachment, and relationship quality. Cyberpsychology, Behavior, and Social Networking, 2, 71-74. doi:10.1089/cyber.2009.0409.

Givertz, M., \& Segrin, C. (2012). The association between overinvolved parenting and young adults' self-efficacy, psychological entitlement, and family communication. Communication Research, 4, 111-1136. doi:10.1177/0093650212456392

Gonçalves, M. I., \& Barros, L. (2008). Inventário de preocupações e valorizações para jovens: Contributo para a avaliação das atribuições de preocupação e importância a áreas da vida de jovens na fase de transição para a adultícia. $R$ Revista Iberoamericana de Diagnóstico y Evaluación - e Avaliação Psicológica, 26, 141-166.

Gronlick, W. S., \& Ryan, R. M. (1989). Parent styles associated with children's selfregulation and competence in school. Journal of Educational Psychology, 81, 143-154.

Hofer, B. K., \& Moore, A. S. (2010). The $i$ connected parent: Staying close to your kids in college (and beyond) while letting them grow up. New York: Free Press.

Hoghughi, M. (2004) Parenting: An introduction. In M. Hoghughi \& N. Long (Eds), Handbook of parenting: Theory and research for practice (pp. 1-18). London: Sage.

Holden, G. W. (2010). Parenting: A dynamic perspective. Thousand Oaks, CA: Sage.
Howe, N., \& Strauss, W. (2003). Millennials go to college. Washington, DC: American Association of Collegiate Registrars and Admissions Officers (AACRAO) and Life Course Associates.

Ingen, D., Freheit, S., Steindeldt, J., Moore, L., Wimer, D., Knutt, A., Scapinello, S., \& Roberts, A. (2015). Helicopter Parenting: The effect of an overbearing caregiving style on peer attachment and self-efficacy. Journal of College Counseling, 18, 7-20.

Jablonski, J., \& Martino, S. D. (2013). A qualitative exploration of emerging adults' and parents' perspectives on communicating adulthood status. The Qualitative Report, 18(37), 1-12.

Kenyon, D. B., \& Koerner, S. S. (2009). Examining emerging adults' and parents' expectations about autonomy during the transition to college. Journal of Adolescent Research, 24, 293-320.

Koerner, A. F., \& Fitzpatrick, M. A. (2002). Toward a theory of family communication. Communication Theory, 12, 70-91. doi:10.1093=ct=12.1.70

Luyckx, K., Soenens, B., Vansteenkiste, M., Goossens, L., \& Berzonsky, M. (2007). Parental psychological control and dimensions of identity formation in emerging adulthood. Journal of Family Psychology, 21(3), 546550.

Kane, M. (2005). Contemporary issues in parenting. New York: Nova Science Publishers.

Kublikowski, I., \& Rodrigues, C. M. (2016). "Kangaroo generations": New contexts, new experiences. Estudos de Psicologia (Campinas), 33(3), 535-542.

LeMoyne, T., \& Buchanan, T. (2011). Does "hovering" matter? Helicopter parenting and its effect on well-being. Sociological Spectrum, $\quad 31, \quad 399-418$. doi:10.1080/02732173.2011.574038

Locke, J., Campbell, M. A., \& Kavanagh, D. J. (2012). Can a parent do too much for their child? An examination by parenting professionals of the concept of overparenting. Australian Journal of Guidance and Counselling, 22, 249-265. 
Luyckx, K., Schwartz, S. J., Berzonsky, M. D., Soenens, B., Vansteenkiste, M., Smits, I., \& Goossens, L. (2008). Capturing ruminative exploration: Extending the four-dimensional model of identity formation in late adolescence. Journal of Research in Personality, 42, 58-82.

Maccoby, E. E., \& Martin, J. (1983). Socialization in the context of the family: Parentchild interaction. In M. Hetherington, $\mathrm{H}$, \& Mussen (Eds). Handbook of child psychology, 4, Socialization, personality and social development (pp. 1-101). New York: Wiley.

Maccoby, E. (2000). Parenting and its effects on children: On reading and misreading behavior genetics. Annual Review of Psychology, 51, 127.

Marano, H. E. (2004). A nation of wimps. Psychology Today, 58-70.

Marôco, J. (2007). Análise estatística - Com utilização do SPSS ( $3^{\mathrm{a}}$ Ed.). Lisboa: Edições Sílabo, Lda.

Marôco, J. (2010). Análise de equações estruturais - Fundamentos teóricos, software e aplicações. Pêro Pinheiro: ReportNumber, Lda.

Marôco, J. (2014). Análise estatística - Com utilização do SPSS (6 ${ }^{\mathrm{a}}$ Ed.) Pêro Pinheiro: ReportNumber, Lda.

Mendonça, M., Andrade, C., \& Fontaine, A. M. (2009). Transição para a idade adulta e adultez emergente: Adaptação do questionário de marcadores de adultez junto de jovens portugueses. Psychologica, 51, 147-168.

Meyer, B., Enstrom, M. K., Harstveit, M., Bowles, D. P., \& Beevers, C. G. (2007). Happiness and despair on the catwalk: Need satisfaction, well-being, and personality adjustment among fashion models. The Journal of Positive Psychology, 2, 2-17.

Montandon, C. (2005). As práticas educativas parentais e a experiência das crianças. Educação \& Sociedade, 26(91), 485-507.

Montgomery, N. (2010). The negative impact of helicopter parenting on personality. In Poster session presented at the annual meeting of the Association of Psychological Science, Boston, MA.

Odenweller, K., Booth-Butterfield., \& Weber, K. (2014). Investigating helicopter parenting, family environments, and relational outcomes for millennials. Communication Studies, 65, 407-425.

Padilla-Walker, L. M., \& Nelson, L. J. (2012). Black hawk down? Establishing helicopter parenting as a distinct construct from other forms of parental control during emerging adulthood. Journal of Adolescence, 35, 11771190. doi:10.1016/j.adolescence.2012.03.007

Petrogiannis, K. (2011). Conceptions of the transition to adulthood in a sample of Greek higher education students. International Journal of Psychology and Psychological Therapy, 11(1), 121-137.

Perris, C., Jacobsson, L., Lindström, H., Von Knorring, L. \& Perris, H. (1980). Development of a new inventory for assessing memories of parental rearing behaviour. Acta Psychiatrica Scandinavica, 61, 265-274. doi:10.1111/j.1600-0447.1980.tb00581.x

Prioste, A., Lugar, A., Paulino, P., Jongenelen, I., \& Rosa, P. J. (2018). Escala das Dimensões do Desenvolvimento da Identidade: Estudos psicométricos iniciais. Psicologia, 32(2), 114. doi:10.17575/rpsicol.v32i2.1244

Prioste, A., Tavares, P., \& Magalhães, E. (2019). Tipologias de funcionamento familiar: Do desenvolvimento identitário à perturbação emocional na adolescência e adultez emergente. Análise Psicológica, 2(XXXVII), 173-192. doi:10.14417/ap.1534

Rainey, A. (2006). Survey provides further evidence of high parental involvement with college students. Higher Education Journal, 39-43.

Reader, P., Duncan, S., \& Lucey, C. (2005). Studies in the assessment of parenting. Florence: Routledge.

Reed, K., Duncan, J., Lucier-Greer, M., Fixelle, C., \& Ferraro, A. (2016). Helicopter parenting and emerging adult self-efficacy: implications for mental and physical health. Journal of Child and Family Studies, 25, 3136-3149.

Relvas, A. P. (2006). O ciclo vital da família Perspectiva Sistémica (4 ${ }^{\mathrm{a}}$ Ed.). Porto: Edições Afrontamento.

Ryan, R. M., \& Deci, E. L. (2000). Selfdetermination theory and the facilitation of intrinsic motivation, social development, and 
wellbeing. American Psychologist, 55, 68-78. doi:10.1037110003-066X.55.1.68

Scabini, E., Marta, E., \& Lanz, M. (2006). The transition to adulthood and family relations: An intergenerational perspective. London: Psychology Press.

Schiffrin, H. H., Godfrey, H., Liss, M., \& Erchull, M. J. (2015). Intensive parenting: Does it have the desired impact on child outcomes? Journal of Child and Family Studies, 24, 2322-2331. doi:10.1007/s10826-014-0035-0

Schiffrin, H. H., Liss, M., Miles-McLean, H., Geary, K. A., Erchull, M. J., \& Tasher, T. (2014). Helping or hovering? The effects of helicopter parenting on college students' wellbeing. Journal of Child and Family Studies, 23, 548-557. doi:10.1007/s10826-013-9716-3.

Segrin, C., Givertz, M., Swaitkowski, P., \& Montgomery, N. (2015). Overparenting is associated with child problems and a critical family environment. Journal of Child and Family Studies, 24, 470-479.

Segrin, C., Woszidlo, A., Givertz, M., \& Montgomery, N. (2013). Parent and child traits associated with overparenting. Journal of Social and Clinical Psychology, 32, 569595. doi:10.1521/jscp.2013.32.6.569.

Segrin, C., Woszidlo, A., Givertz, M., Bauer, A., \& Murphy, M. (2012). The association between overparenting, parent-child communication, and entitlement and adaptive traits in adult children. Family Relations, 61, 237-252. doi:10.1111/j.1741-3729.2011.00689.x.

Sijtsma, K. (2012). Future of psychometrics: Ask what psychometrics can do for psychology. Psychometrika, 77(1), 4-20.

Spokas, M., \& Heimberg, R. G. (2009). Overprotective parenting, social anxiety, and external locus of control: Cross-sectional and longitudinal relationships. Cognitive Therapy and Research, 33, 543-551. doi:10.1007/s10608-008-9227-5.

Steinberg, L. (2005). Psychological control: Style or substance? New Directions for Child and Adolescent Development, 108: 71-78.

Teodoro, M. L., Land, B. R., \& Allgayer, M. (2009). Desenvolvimento e validade fatorial do Inventário do Clima Familiar (ICF) para adolescentes. Psicologia: Teoria e prática, 11(3), 27-39.

Ungar, M. (2009). Overprotective parenting: Helping parents provide children the right amount of risk and responsibility. The American Journal of Family Therapy, 37, 258-271.

van Ingen, D. J., Freiheit, S. R., Steinfeldt, J. A., Moore, L. L., Wimer, D. J., Knutt, A. D., ..., \& Roberts, A. (2015). Helicopter parenting: The effect of an overbearing caregiving style on peer attachment and self-efficacy. Journal of College Counseling, 18,7-20.

Vansteenkiste, M., Zhou, M. M., Lens, W., \& Soenens, B. (2005). Experiences of autonomy and control among Chinese learners: Vitalizing or immobilizing? Journal of Educational Psychology, 97, 468-483.

Weber, L., Selig, G., Bernardi, M., \& Salvador, A. (2006). Continuidade dos estilos parentais através das gerações: Transmissão intergeracional de estilos parentais. Paidéia, 16(35), 407-414.

Wei, M., Philip, A. S., Shaffer, A., Young, S. K., \& Zakalik, R. A. (2005). Adult attachment, shame, depression, and loneliness: The mediation role of basic psychological needs satisfaction. Journal of Counseling Psychology, 52, 591-601. doi:10.1037/0022-0167.52.4.591 